\title{
Analysis of Pattern of Information Revelation and Site Use Behavior in Social Networking Sites
}

\author{
Sanjeev Dhawan \\ University Institute of Engineering and Technology \\ Kurukshetra University \\ Haryana, India
}

\author{
Shivi Goel \\ University Institute of Engineering and Technology \\ Kurukshetra University \\ Haryana, India
}

\begin{abstract}
With the emergence of online social networking sites, the rules of social interaction and communication has been changed. Most of the social networking sites motivate users to share personal information, build new relationships and increase knowledge with a perceived ease of use. But this online interaction and sharing of personal information on online sites have raised many new privacy concerns as it provide huge amount of data to third party which can be misused by malicious activities against the user's will. This research aims to develop an understanding of individual's risk taking behavior online particularly around the issue of information disclosure and to study the factors influencing the site use behavior. Human behavior concern has crucial role in development of social networking sites. Using an online questionnaire, survey data has been collected from social networking site users of different age groups and gender. It has been statistically shown that although the privacy concerns of respondents are significant but their attitude towards the risks of information disclosure is still very relaxed. People are still not aware about the actual cyber risks and therefore still happy to disregard protective advice and have risk taking behavior. Further some actual flaws and changes that user's wishes to see in online social networking sites are mentioned which are also collected from the actual online interaction with the users.
\end{abstract}

Keywords: Awareness, Information revelation, Privacy concern, Social networking sites (SNS), Social engineering.

\section{INTRODUCTION}

In 1954 J.A. Barnes coined the term 'social Networking' in references to a map of the relationships between individuals, indicating the ways in which they are connected through various social familiarities ranging from casual acquaintances to close familial bonds. After half a century and with the help of internet the basic concept of these social networks has completely changed. In recent years several attractive, user friendly and cost effective facilities has been introduced in online society in which social networking sites are very popular where an extensive participation of people has been seen. Boyd and Ellison defined social networking sites as web-based services that allow individuals to "(1) construct a public or semi-public profile within a bounded system, (2) articulate a list of other users with whom they share a connection, and (3) view and traverse their list of connections and those made by others within the system" [1]. Social networking sites provide a platform to stay connected with friends, family, to develop new connections and share personal information and experience.

Social networking methods were came into focus by the end of 1990's. The new generation of social networking began to gain momentum with the surfacing of Friendster in 2002 followed by Myspace and LinkedIn a year later. Facebook launched in 2004, and now become the most popular site in the world [2]. According to rough statistics as on September 2013 [3], Facebook have 727 million daily active users on average. The phenomenon of online social interaction is more than teenagers looking to expand their friend list or to gain popularity. People of all age group and demographics are engaged in enriching their lives through the contacts they make on social networking sites. Even it provides a wide platform for marketing and advertisement agencies to promote their products.

As users can share almost anything with anybody on online social networking sites, social networking sites provide many privacy policies to control and customize what their information is available to other users. However it has been shown [4] [5] that such measures are not enough to protect one's sensitive data. Social ties represented as network data can be demoralized by the challenger to predict the value of sensitive attributes through a wide array of techniques.

The rapid growth of social networking sites has attracted a large number of researchers to explore and study its popular services. This paper focuses on the study and analysis of use behavior of these sites, user's attitude towards privacy and security in non invasive manner, to develop an understanding of individual's risk taking behavior online particularly around the issue of information revelation. Rest of the paper is organized as follows. Section 2 elaborates the theoretical background, section 3 presents the data analysis and results of the paper and section 4 concludes the work.

\section{THEORITICAL BACKGROUND}

The role of peer pressure, herding behavior, myopic privacy attitude, sense of protection offered by SNS has been clarified by Gross et al. which support the information revelation behavior of users and quantified individual's willingness to provide large amount of personal data and expose them to various physical and cyber risks [6]. In terms of perceived trust and privacy concern, subjects from Facebook and Myspace are compared in order to study the use behavior and it has been shown that the interaction of trust and privacy concern in SNS is not yet fully understood to sufficient degree by users to allow accurate modeling of behavior and activity [7]. Steinfield et al. explains the impact of self esteem on use of SNS and demonstrated that lower self esteem students appear to gain more from their use of Facebook than higher self esteemed students as SNSs mitigates fear of rejection and provide ease to facilitate initial communication [8]. Role of national cultural differences in user adoption of SNS is investigated by Veltri et al. which specially focus on Morocco and U.S. users [9]. It uses Hof stede's dimensions of national culture and diffusion of innovation. Phippen et al. demonstrated that users usually get engaged in risk taking behavior and are willing to disclose potentially harmful information to strangers [10]. Gangadharbatla et al. investigated that internet self efficacy, need to belong and 
collective self esteem, all have positive effects on attitude of user towards SNS [11]. The factors that drive students to use SNS are explored by Cheung et al. [12]. They conceptualized the use of SNS as an intentional social action and demonstrated that social presence is an important factor that determines student's usage of Facebook. Even Purcell et al. demonstrated that the impact of Facebook is more than Twitter on teens [13]. Robben et al. proposed a model which can help in determining the impact of social learning technologies in workplace. It emphasizes on fundamental trust which results in knowledge productivity and enhanced social capital [14]. The impact of trust, security and privacy concern with regard to social networking websites among consumers using both reliable scale and measures are examined by Shin et al. In this paper it is suggested that SNS providers need to establish a trust relationship with consumers by developing and promoting comprehensive standards and ensuring that participants of privacy seal programs remain to those standards [15]. Lin et al. suggested that the influence on user intention to join SNS, enjoyment has stronger significant role on people's continued use of SNS [16]. It also demonstrated that gender makes a notable difference in the effect of perceived benefit indicating that men do not feel pleasure with SNS with a large number of members. Qin et al. suggested that perceived ease of use, perceived usefulness and social influence positively relate to usage intention of SNSs [17]. Tsoi et al. presented cultural comparison of the impact of privacy concern on the use behavior of SNS and revealed that under the influence of privacy concern, usage behavior on same SNS are not identical [18]. Alam et al. reported the results of an investigation of the use of Facebook by the Australian taxation office [19]. In order to understand the impact of user's privacy concern on their acceptance of SNS, Xin Tan et al. demonstrated two research models with privacy concern conceptualized either as an antecedent of acceptance intention or as a moderator of the relationship in the technology acceptance model. It is statistically shown that privacy concern did restrain the effect of perceived usefulness and perceived ease of use on user's intention to continue the use of SNS [20]. Quinn et al. demonstrates the impact of age difference on the use behavior of SNSs [21]. Dhami et al. proposed a research model which advocates that perceived security, perceived privacy and perceived trust are the factors that influence user's willingness to share information in SNSs [22]. The research model proposed by Mohamed et al. explained that self efficacy, perceived severity, perceived vulnerability and response efficacy are positively related to information revelation [23]. The research model proposed by Chen et al. advocated that social presence, ease of use and personality traits such as extroversion contributes to perceived enjoyment which positively contributes to site use. The personal beliefs of the computing environment such as internet risk perception and privacy abuse concern will impact on one's perceived risk which in turn negatively relate to site use behavior [24]. Elaborated study of personality and its effects on social media is demonstrated by Correa et al. where personality traits like extraversion, emotional stability and openness to experience is discussed [25]. User behavior is studied from four different perceptive which are connection and interaction, traffic activity, mobile social behavior and malicious behavior [26]. As SNS can be used as a tool of customer engagement, social interaction and relationship building, constantinides et al. investigated the use of SNS as a business tool [27].

\section{DATA ANALYSIS AND RESULT}

Data is obtained through the online based questionnaire. It has been statistically shown that although the privacy concerns of respondents are significant but their attitude towards the risks of information disclosure is still very relaxed. Figure 1 shows the attitude of social networking site users towards the potential risks. $30 \%$ of the total respondents do not care about the risks in spite of having the knowledge of misuse of personal information. Figure 2 shows the level of understanding of privacy settings and terms and conditions. Analysis shows that only $36 \%$ claims to have full understanding of these settings and agreements. Figure 3 shows the usage trend of social networking sites. Analysis shows that $49 \%$ respondents use these sites for sharing information. Figure 4 shows the reaction of users when asked would they stop using SNS if SNS continued to use their personal information and photographs with researchers and marketers against user's expectations. 52\% users confirmed that they would definitely stop using such sites.

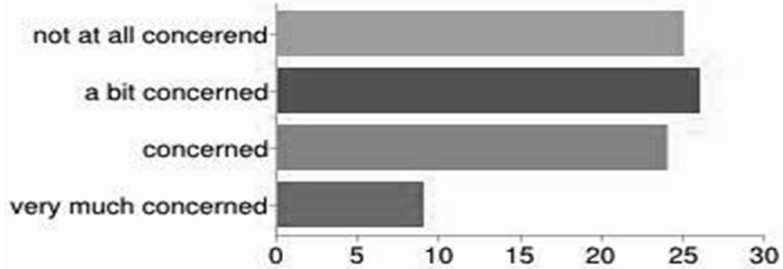

Fig. 1: attitude of users towards privacy concern

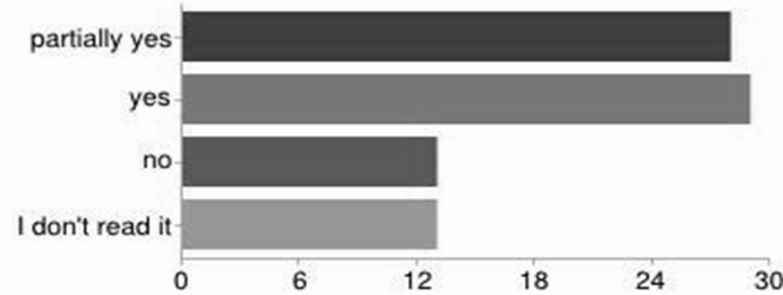

Fig. 2: level of understanding of privacy settings and terms and conditions

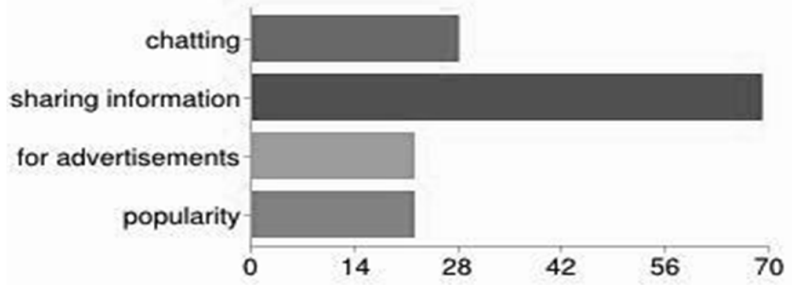

Fig. 3: usage trend

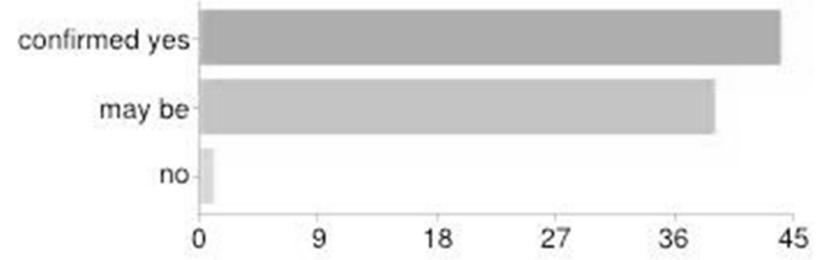

Fig. 4: user's response about using SNS under influence of unwanted information disclosure

It is analyzed that SNS user especially Indian citizen have mixed feelings on privacy issues. Their perceptions regarding privacy issues are still not clear and it is supposed that it is because of lack of awareness. As shown in above graphs that $52 \%$ users agree to stop using SNS if they found misusage of 
their private information but still $30 \%$ of total respondents do not actually care about this issue.

\section{CONCLUSION}

The primary objective of this study is to examine the affect of privacy issues on site use behavior in social networking sites. Site use adds vital support to the sustainable success of networking sites and may simulate the use extent by cultivating perceived hedonic utilities. Some of the flaws and changes in terms of security and privacy as suggested by respondents of online survey are like the message receiving mechanism should be scrutinized again. There should be some control like id proof verification on fake ids. Term and condition page and privacy settings page are often too long and confusing. These pages should be aided with less text and more graphical representations to increase the understanding of the user. Also Social networking sites can provide an option of user defined security settings where user's will be allowed to adjust the privacy settings according to their needs not just according to prearranged format provided by online social networking site providers. In future detailed in depth analysis will be done to study the site use behavior in social networking and impact of privacy concern on it.

\section{REFERENCES}

[1] D.M. Boyd, N.B. Ellison, Social network sites: definition, history, and scholarship, Journal of Computer-Mediated Communications 13 (1) (2007) 210-230

[2] 2014 Best Social Networking Site reviews and Comparisons at: http://social-networking-websitesreview.toptenreviews.com/index.html

[3] Statistics at: http://newsroom.fb.com/Key-Facts

[4] J. He, W. Chu, and Z. Liu, "Inferring privacy information from social networks," Intelligence and Security Informatics, pp. 154-165, 2006

[5] E. Zheleva and L. Getoor, "To join or not to join: the illusion of privacy in social networks with mixed public and private user profiles," in Proc. of ACM World Wide Web, 2009, pp. 531-540.

[6] Gross, Ralph, and Alessandro Acquisti, "Information revelation and privacy in online social networks," in Proceedings of the 2005 ACM workshop on Privacy in the electronic society, ACM, 2005.

[7] Dwyer, Catherine, Starr Roxanne Hiltz, and Katia Passerini, "Trust and Privacy Concern Within Social Networking Sites: A Comparison of Facebook and MySpace" AMCIS. 2007.

[8] Steinfield, Charles, Nicole B. Ellison, and Cliff Lampe, "Social capital, self-esteem, and use of online social network sites: A longitudinal analysis."Journal of Applied Developmental Psychology 29.6 (2008): 434-445.

[9] Veltri, Natasha F. and Wafa Elgarah, "The role of national cultural differences in user adoption of social networking" in Southern Association for Information Systems Conference, Charleston, SC, 2009.

[10] Phippen, Andy, Richard Davey, and Steven Furnell, "Should we do it just because we can? Methodological and ethical implications for information revelation in online social networks" in Methodological Innovations Online 4.3 (2009): 41-55.

[11] Gangadharbatla, Harsha, "Facebook me: Collective selfesteem, need to belong, and internet self-efficacy as predictors of the iGeneration's attitudes toward social networking sites" in Journal of interactive advertising 8.2 (2008): 5-15.
[12] Cheung, Christy MK, Pui-Yee Chiu, and Matthew KO Lee, "Online social networks: Why do students use Facebook?" in Computers in Human Behavior27.4 (2011): 1337-1343.

[13] Purcell, Kristen, Aaron Smith, and Kathryn Zickuhr, "Social media \& mobile internet use among teens and young adults" in Washington, DC: Pew internet \& american life project, 2010.

[14] Robben, Joost, "A model for leveraging social learning technologies in corporate environments" in Proceedings of Network Learning Conference. 2010.

[15] Shin, Dong-Hee, "The effects of trust, security and privacy in social networking: A security-based approach to understand the pattern of adoption" in Interacting with Computers 22.5 (2010): 428-438.

[16] Lin, Kuan-Yu, and Hsi-Peng Lu, "Why people use social networking sites: An empirical study integrating network externalities and motivation theory," in Computers in Human Behavior 27.3 (2011): 1152-1161.

[17] Qin, Li, Yongbeom Kim, Jeffrey Hsu, and Xin Tan, "The effects of social influence on user acceptance of online social networks" in International Journal of Human-Computer Interaction 27, no. 9 (2011): 885-899.

[18] Tsoi, Ho Keung, and Li Chen, "From Privacy Concern to Uses of Social Network Sites: A Cultural Comparison via User Survey" in Privacy, security, risk and trust (passat), 2011 ieee third international conference on and 2011 ieee third international conference on social computing (socialcom), pp. 457-464. IEEE, 2011

[19] Alam, Sultana Lubna, John Campbell, and Richard Lucas, "Using Social Media in Government: The Australian Taxation Office e-Tax Facebook Page."Dependable, Autonomic and Secure Computing (DASC), 2011 IEEE Ninth International Conference on. IEEE, 2011

[20] Xin Tan, Li Qin, Yongbeom Kim, Jeffrey Hsu, "Impact of privacy concern in social networking web sites", Internet Research, Vol. 22 Iss: 2, 2011, pp.211 - 233

[21] Quinn, Darren, Liming Chen, and Maurice Mulvenna. "Does Age Make a Difference in the Behaviour of Online Social Network Users?" in International Conference on Cyber, Physical and Social Computing, IEEE, 2011.

[22] Dhami, Anil, et al. "Impact of trust, security and privacy concerns in social networking: An exploratory study to understand the pattern of information revelation in Facebook" in IEEE, 2012.

[23] Mohamed, Norshidah, and Ili Hawa Ahmad, "Information privacy concerns, antecedents and privacy measure use in social networking sites: Evidence from Malaysia" in Computers in Human Behavior (2012).

[24] Chen, Rui, "Member use of social networking sites-an empirical examination" in Decision Support Systems 54.3 (2013): 1219-1227.

[25] Correa, Teresa, et al, "Personality and Social Media Use", 2013.

[26] Jin, Long, Yang Chen, Tianyi Wang, Pan Hui, and Athanasios V. Vasilakos, "Understanding user behavior in online social networks: A survey." IEEE Communications Magazine 2013

[27] Constantinides, Efthymios, and Carlota Lorenzo-Romero, "Social Networking Sites as Business Tool: A Study of User Behavior" in Business Process Management, pp. 221-240, Springer Berlin Heidelberg, 2013. 